\title{
INTEGRATED STUDY ON THE VELOCITY FIELD INDUCED BY PLUNGING BREAKERS
}

\author{
Germán Rivillas-Ospina ${ }^{1}$, Adrián Pedrozo-Acuña ${ }^{1}$, Rodolfo Silva ${ }^{1}$, Alec Torres-Freyermuth ${ }^{2}$ \\ and Cesar Gutierrez ${ }^{1}$
}

\begin{abstract}
In this investigation we employ a technique called Bubble Image Velocimeter (BIV) to obtain measurements of flow fields in the surf-zone. This technique allows measurements of flow velocity in the aerated breaking zone, where other techniques are ineffective. The technique has been widely used to study flow propagation in front of vertical structures, it is employed in this study to estimate the velocity field induced by the propagation of a plunging wave travelling over an impermeable slope. The BIV measurements were compared to those obtained with an Acoustic Doppler Velocimeter (ADV) showing that the BIV technique is more suitable when the velocity field is estimated under the presence of an aerated flow. Moreover, the phase-averaged velocity fields obtained from the numerical model were compared against those evaluated from the BIV measurements. A reliable estimation of the VOF-type numerical derivations in the surf zone was established. In the swash zone, an over prediction of the offshore flow was identified.
\end{abstract}

Keywords: BIV; ADV; RANS, VOF; Modelling

\section{INTRODUCTION}

The Acoustic Doppler Velocimeter (ADV) has been used to study wave velocities and turbulent flows. In recent years these methods have been widely employed in the study of turbulence in the surf zone, in estuarine and coastal environments (Scott et al. 2005) and in the understanding of feedback processes in the nearshore zone (Raubenheimber et al. 2004). However ADV measurements in this zone have the limitation that they can present significant "noise" and inaccurate velocity measurements due to the presence of the air bubbles (Elgar et al. 1994). Therefore, non-intrusive techniques have been employed to measure the flow properties in aerated flows, such Particle Image Velocimetry (PIV). This technique was successfully applied for the measurements of flow fields in the surf zone (Govender et al. 2004), which is the base of this work and is the continuation of previous research in the analysis of wave propagation under plunging breakers (Rivillas et al. 2011) that shows an investigation into the spatial and temporal evolution of the velocity field induced by plunging waves using the Bubble Image Velocimetry (BIV) technique.

Due to the lack of an instrument that provides flow information in the presence of air bubbles, we employ a technique called bubble image velocimetry (BIV) (Ryu et al 2005) which allows the measurements of flow velocity within this zone, where PIV is ineffective. The BIV technique has been successfully used in previous studies to measure the velocity field in green water and in the aerated region of the breaker close to vertical structures (Ryu and Chang 2008; Ryu et al 2005, 2007), as well as overtopping events from violent wave impacts (Jayaratne et al 2008).

The results of the BIV technique are compared with both the Acoustic Doppler Velocimeter methodology and those obtained from a VOF model based on the Reynold Navier-Stokes (RANS) equations. The comparison evaluates the transient two-dimensional nature of the flow field, assessing the behavior of the wave transformation and leading to understanding of the spatio-temporal velocity field within the analyzed zone.

\section{METHODOLOGY}

\section{Experimental Set-up}

The experiments were performed in the two-dimensional wave flume of the Coastal Engineering Laboratory of at the National University of Mexico (Fig. 1). The experimental data includes detailed

\footnotetext{
${ }^{1}$ Instituto de Ingeniería, Universidad Nacional Autónoma de México, Cd. Universitaria, 04360, México

2 Instituto de Ingeniería, Universidad Nacional Autónoma de México, Puerto de Abrigo s/n, 92718, Sisal, México
} 
velocity measurements along the impermeable slope. The mean water depth was set to $h=0.44 \mathrm{~m}$. The experimental programme included detailed velocity measurements for two selected waves with a period of 1.5 seconds: a) wave height $H=0.18 \mathrm{~m}$ and b) wave height $H=0.10 \mathrm{~m}$. The velocity point measurements for the comparison were taken using an ADV (Fig. 4) at different positions on the slope (offshore, breaking and swash zones). The wave conditions are based on the value of their associated Iribarren number that comprises 0.6 and 1.017 (Pedrozo-Acuña et al. 2008). These values produce more air in the fluid due to the strong turbulence caused by the plunging breaker within these conditions, hence the application of the BIV technique.

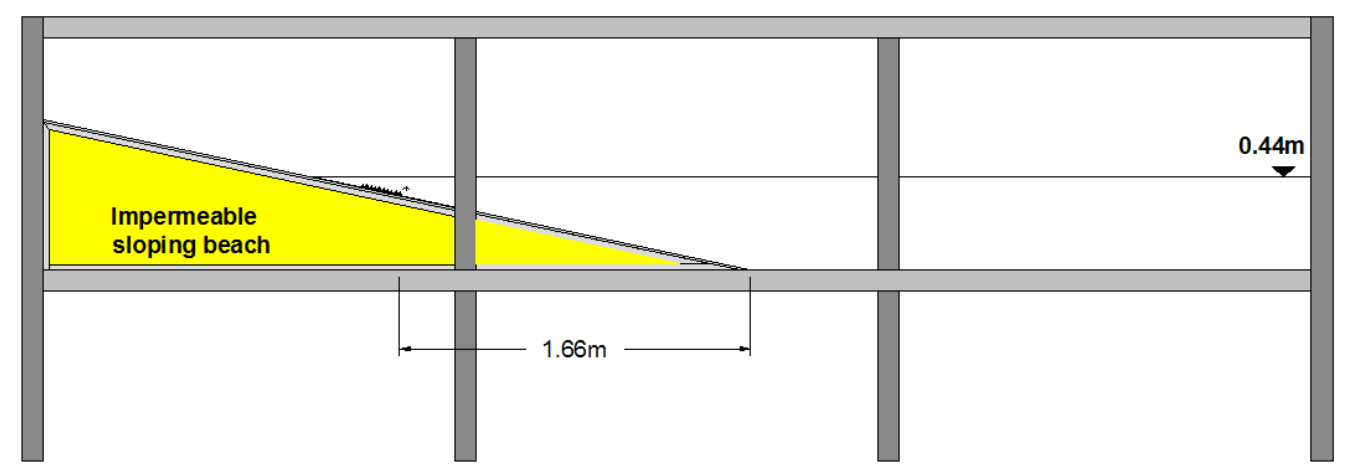

Figure 1 Two dimensional wave flume of the National University of Mexico

The equipment used in the experimental setup comprises a high-speed digital video camera, the Fresnel lighting of $650 \mathrm{~W}$ at the back corners and an open face light placed above the impermeable beach slope (Fig. 2). The video recording was carried out at a rate of $1.008 \mathrm{fps}$ with a resolution of $1248 \times 554$ for a single wave period. The aperture of the camera was set with an f-number between 5.6 and 8.0, and the distance $\mathrm{L}$ was $1.33 \mathrm{~m}$. The camera was placed perpendicular to the glass side wall of the flume.

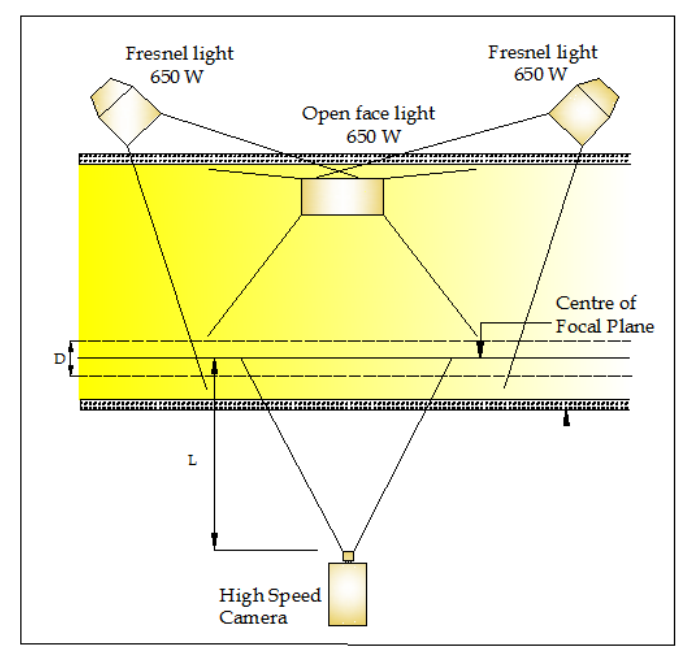

Figure 2 Experimental setup of the BIV technique

The evolution of the turbulent process is a result of the strong flux due to the wave breaking over the impermeable slope. The velocity fields within eddies and the recirculation flow are estimated through cross-correlating the images obtained with the video camera, in the same way that the PIV technique examines the spatial dependent turbulent velocity components. The seeding particles in this technique are the bubble structure captured by the video camera, using the same procedure employed in the PIV system. Many consecutive images are taken for the cross-correlation algorithm by employing the 
shawdography technique, which allows better identification of the air in the snapshots (Fig. 3). This system has been applied to study flow in stratified liquids, for evaluation of the velocity field in two phase flows and in the cross-correlation algorithms derived from PIV methodologies (Hassan et al 1998, Nishino et al 2000, Lindken and Merzkirch 2002). The Minimum Quadratic Difference (MQD) algorithm, based on a least-squared technique was used for the determination of the velocity vectors. This method follows the tracks of patterns comprised by particles in consecutive images.
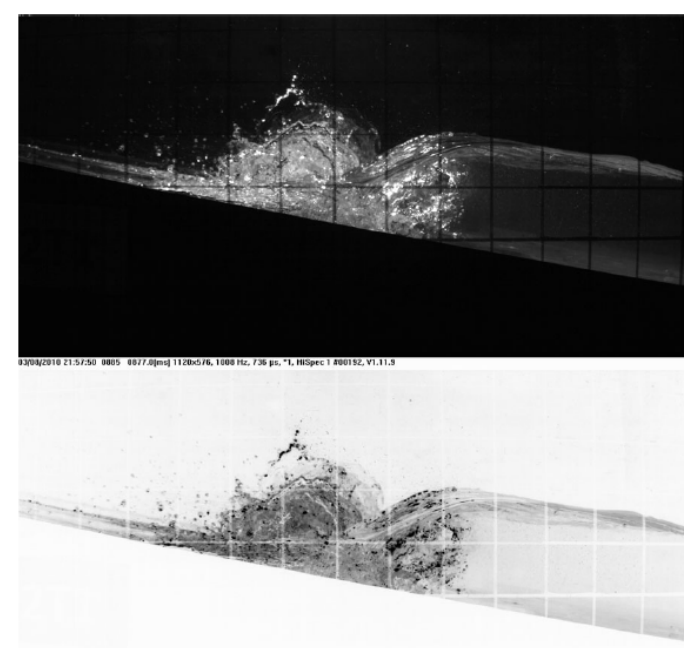

Figure 3 Example of Shawdography technique for a plunging wave

For the estimation of the phase-averaged velocity components consecutive video images were required, from which 20 repetitions of the instantaneous velocity measurements of the BIV results were constructed. In the utilization of the BIV technique, a limiting depth of field (DOF) was defined. This concept represents the focal distance of the camera, where objects appear sharp and well-focused. The field of view (FOV) was determined assuming that the lens focuses on a point at a distance $\mathrm{L}$ from the nodal point of the lens. For the experiments presented here, the FOV was 70x30 cm $(1245 \times 505 \mathrm{~cm})$. The DOF was estimated by means of the formulae proposed by Ray (2002), which can be expressed as follows:

$$
D O F=S-R
$$

where $\mathrm{R}$ represents the nearest limit and $\mathrm{S}$ the farthest limit of the DOF. The estimations of these two edges $(S$ and $R$ ) were undertaken following

$$
\begin{aligned}
& S=\frac{L f^{2}}{\left(f^{2}-N L C\right)} \\
& R=\frac{L f^{2}}{\left(f^{2}-N L C\right)}
\end{aligned}
$$

In the above equations, $f$ is the focal length of the camera focal lens, $C$ represents the value for the circle of confusion that depends on the property of the camera $(C=0.01)$ and $N$ is the $f$ number of the camera aperture. Bubbles located outside the region defined by the DOF appear blurred in the captured image, producing an unclear texture, which has a small effect on the correlation used for the determination of the velocity field. The bubbles within the DOF appear sharp, with a clear pattern 
resulting from the flow. This pattern was employed in the correlation method for the estimation of the resulting velocity vectors.

Due to the highly turbulent nature of the breaking process generated by a plunging wave, the instantaneous images do not perfectly match the mean velocities in some instants. Thus, the initial time for each event was defined as the instant when the curled free surface of the wave first approached the impermeable slope. This procedure minimizes the errors in the ensemble average which are due to the mismatching of cases.

\section{Numerical Modeling}

The numerical model employed in this study is a phase and depth resolving model which solves the averaged Reynolds Equations and was developed by Liu (1998a, b). The spatially averaged NavierStokes equations are

$$
\begin{gathered}
\frac{\partial\left\langle u_{i}\right\rangle}{\partial x_{i}}=0 \\
\frac{\partial\left\langle u_{i}\right\rangle}{\partial t}+\left\langle u_{j}\right\rangle \frac{\partial\left\langle u_{i}\right\rangle}{\partial x_{j}}=-\frac{1}{\rho} \frac{\partial\left\langle p_{0}\right\rangle}{\partial x_{i}}+g_{i}+\frac{1}{\rho} \frac{\partial\left\langle\tau_{i j}\right\rangle}{\partial x_{j}}-\frac{\partial\left\langle u^{\prime \prime} \partial u^{\prime \prime}\right\rangle_{i}}{\partial x_{j}}
\end{gathered}
$$

where the vectorial quantities $u$ (velocity) and $p_{0}$ (pressure) are decomposed into spatially averaged components $\langle "\rangle$, i.e., $u_{i}=\left(\left\langle u_{i}\right\rangle+u_{i} "\right) / n$ and $p_{0}=\overline{p_{0}}+p_{0} "$. The subscripts denote horizontal $i, j=1$ and vertical $i, j=2$ components, and $v$ is the molecular viscosity. The second term on the right-hand side of (5) is the viscous stress tensor of the mean flow, and the last term of (5) is the correlation of the spatial velocity fluctuation.

The numerical model was chosen to validate the BIV technique with the spatio-temporal snapshots of the velocity field derived by the model. The domain of interest covers the process of the plunging wave impinging on the bed, the transition zone between the surf and the swash zones. The numerical model has the limitation that it does not consider the air entrainment during the wave breaking. Thus, good agreement between model predictions and observations should not be expected. The objective of this comparison is to ensure that the numerical model is able to capture the wave transformation on the sloping beach. The computational domain is composed by spatial discretization with increments of $\Delta x=0.005 \mathrm{~m}$ and $\Delta z=0.005 \mathrm{~m}$. The comparison between the numerical model and the BIV results was carried out at two points on the sloping beach, one in the surf zone and the other in the swash zone.

\section{Validation of the BIV Technique}

Fig. 4 compares the temporal evolution of horizontal velocity in the surf zone at $h=1.8 \mathrm{~cm}$ from the bed, for a wave condition $h=0.44 \mathrm{~m}, H=10 \mathrm{~cm}$ and $T=1.5 \mathrm{~s}$. The top panel shows the measurements taken with a normal video camera, while the lower panel shows comparison of the ensemble horizontal velocity values obtained from both ADV and BIV measurements against the RANS model results. The experimental time series (ADV and BIV) are illustrated along their relative confidence levels evaluated by $\mu \pm \sigma$, where $\mu$ represents the mean value and $\sigma$ is the standard deviation of the measurement. The measurement techniques are in agreement with each other. At the beginning of the time record, just after the passage of the wave crest (from 0 to $0.5 \mathrm{~s}$ ), the uncertainty associated with the ADV measurements is shown to be larger than that determined for the BIV measurements. This is due to the nature of the flow, which at this stage may entrain an important amount of air. At the start of the flow reversal- $t \approx 0.65$ - the error in the ADV is significantly reduced, indicating a better level of confidence than that shown in the BIV measurements. This is a consequence of the reduction of air present in the 
flow, which confirms a better performance of an ADV in flows with a small amount of air. The numerical model results show the same behavior as those observed with both experimental methods and the error bands estimated for the measurements. In particular, the peak onshore velocity is relatively the same in both experimental techniques and the RANS model.

(a)

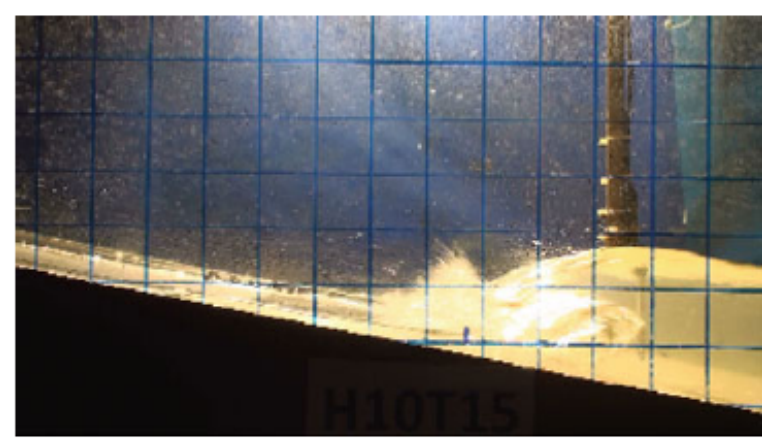

(b)

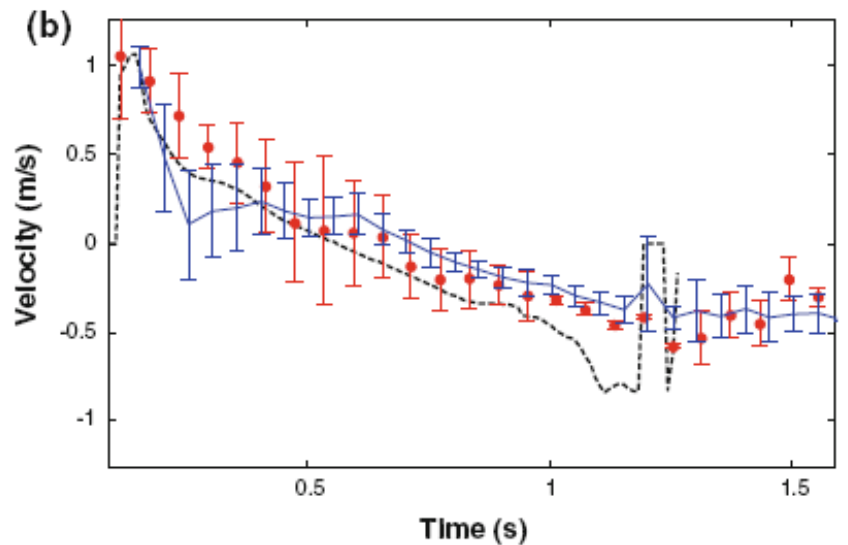

Figure 4 Comparison in the surf zone of the experimental results (BIV-ADV) aganist numerical model. Solid line (ADV), b) dashed line (COBRAS model), c) line filled circle (BIV)

In the Fig 5. the comparison for a point within the swash zone can be seen. At this point the flow is characterised by the run-up of the turbulent bore generated by the wave impinging on the slope. Moreover, the error bars show that BIV measurements are smaller than those depicted for the ADV. This demonstrates the better performance of the BIV technique in this zone, where the turbulence and bubbles are certainly present in the flow. The greater presence of air in the uprush phase of the flow is represented in the large uncertainty associated with the ADV measurements. The RANS model clearly overpredicts the offshore velocity. This is explained by the nature of the numerical model, which does not consider the effects of the air entrainment in the dynamics of the flow.

The averaged velocities obtained with the BIV technique were constructed by repeating 20 events for each selected snapshot. This procedure allows the comparison of the mean vector velocity plots estimated with the numerical model. In Fig 6 the mean velocity vectors are plotted for the uprush phase of the flow, obtained from the BIV technique and those calculated with the RANS model (lower panel). The BIV results show that during the wave impingement over the impermeable slope the splash of water produced from the wave impact can be seen. In addition, this test reveals a vertical structure at the back of the impact point $(x=55 \mathrm{~cm})$. The measurements show that the maximum velocities of the uprush phase are associated with the splash of water, characterized by the mixture of water and air incorporated during the breaking process. 
(a)
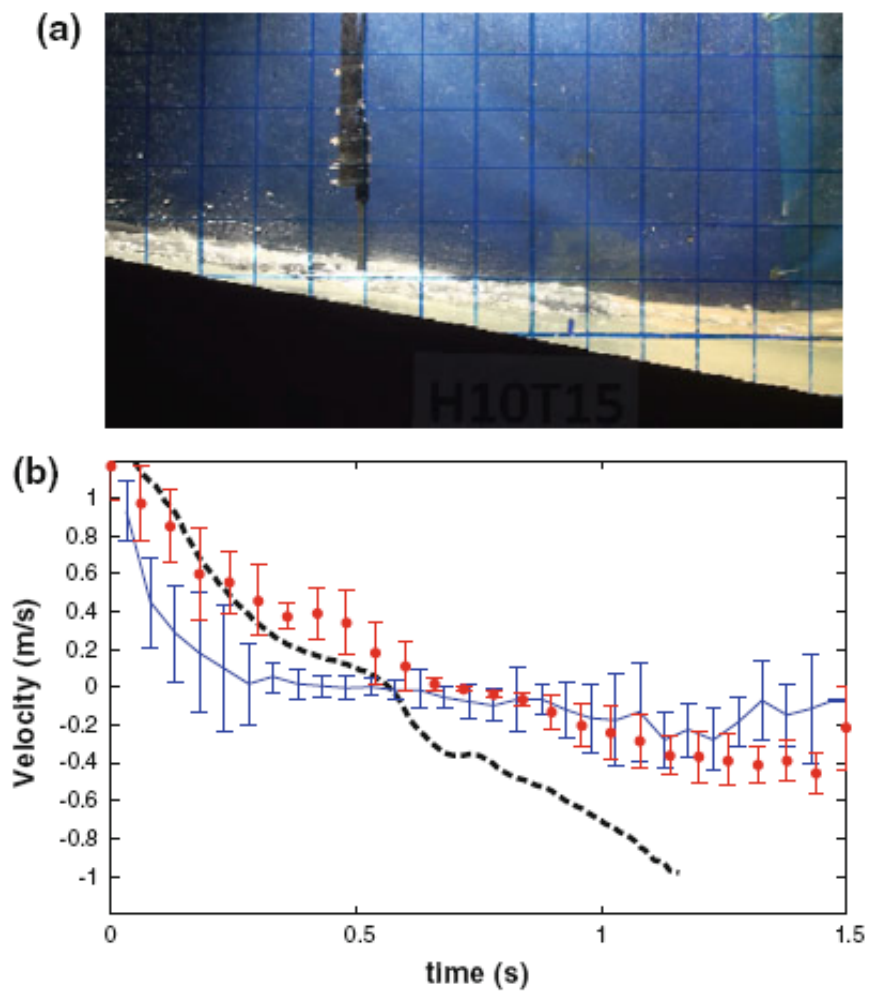

Figure 5 Comparison in the swash zone of the experimental results (BIV-ADV) aganist numerical model. Solid line (ADV), b) dashed line (COBRAS model), c) line filled circle (BIV)
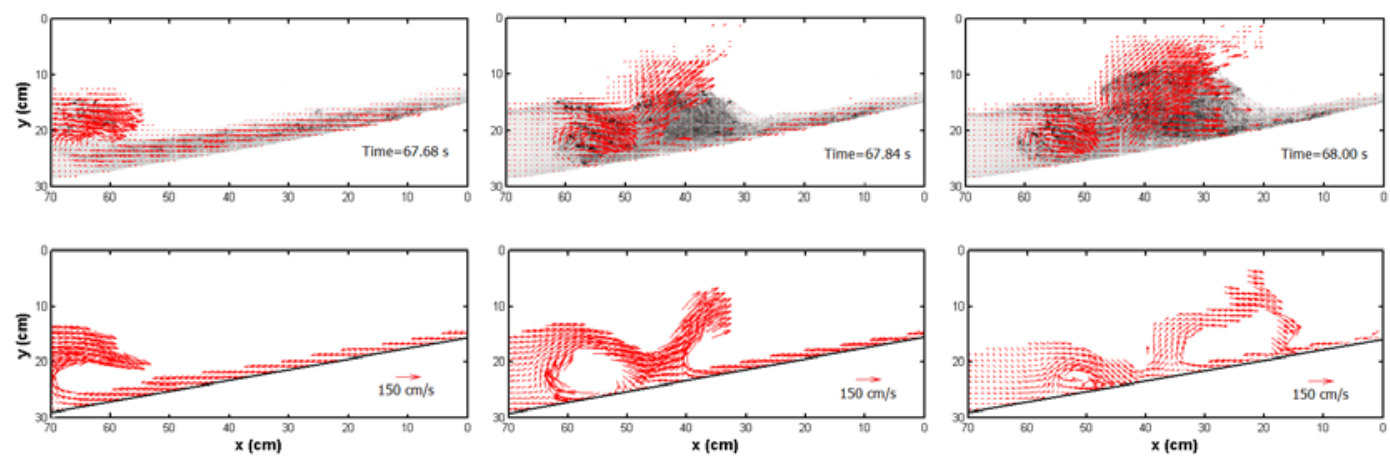

Figure 6 Comparison of the BIV results against the numerical model results for the wave conditions $H=10 \mathrm{~m}$ and $\mathrm{T}=1.5 \mathrm{~s}$

The mean flows in the uprush phase obtained from the numerical simulations exhibit the qualitative behaviour of the flow. The differences arise at the instant of the splash of water, due to the limitations of the turbulence model because its mathematical formulation cannot reproduce biphasic flows.

In Fig. 7 mean quantities during the backwash phase are described. The experimental results show clear predominance of offshore flows with a more uniform flow structure during the backwash against the results given in the uprush phase. The fluid presents a more homogeneous behaviour as result of the turbulent dissipation and the cavitation phenomena. In contrast, the velocity field obtained with the numerical model during this phase reveals better agreement with BIV measurements. But the magnitude of the offshore flows is overestimated by the difference in the representation of air inside the fluid. 

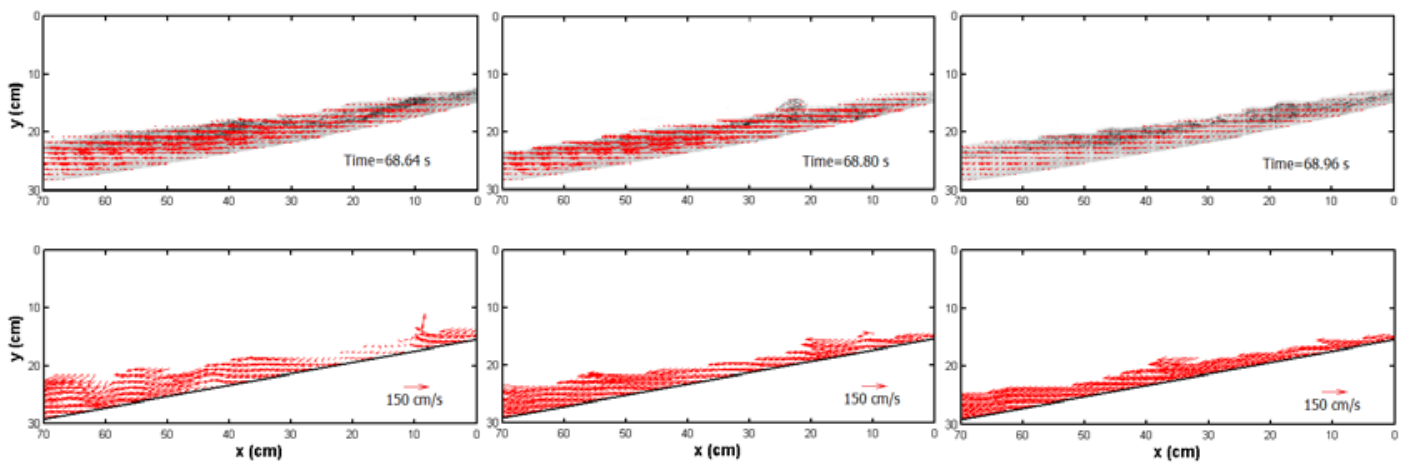

Figure 7 Comparison of the vertical velocity from BIV against the numerical model (RANS) results for the wave conditions $\mathrm{H}=10 \mathrm{~m}$ and $\mathrm{T}=1.5 \mathrm{~s}$

\section{CONCLUSIONS}

This investigation describes the evolution of the velocity field estimated with a non-intrusive method for plunging wave propagation on an impermeable beach slope. The main objective of this study is the validation of the BIV measurements against $\mathrm{ADV}$ results and a comparison with the numerical model simulations. Measurements of the velocity field obtained by bubble image velocimetry (BIV) technique were compared to single point measurements (ADV) along with their relative level of confidence. The comparison of these techniques shows that ADV measurements contain less uncertainty than those derived from BIV due to the slight presence of bubbles in the flow, validating the good behaviour of this technique in this region. The bubbles present in the flow are a consequence of strong turbulence. The times series determined with the numerical model illustrate a similar trend to those obtained with experimental methods. The errors in the BIV technique are smaller than ADV results, indicating a better performance of the BIV in the aerated region.

The numerical model (RANS) was used to evaluate the transient two-dimensional description of the flow field under plunging breakers. In the surf zone, the numerical results show good agreement with respect to the measurements obtained from the BIV, while in the swash zone, the simulations from the model over predict the offshore flow. In addition, the experimental method (BIV) was compared with the RANS model. Good agreement was also found in the overall behaviour of the flow throughout the entire swash event. But in the moment when the wave breaks the numerical model tends to over predict the velocity field with respect to that derived by the BIV technique. Differences between BIV velocity estimations and numerical model predictions can be ascribed to the effects of air compressibility in the dynamics of the resulting jet of water, which indicates the importance of considering the dynamics of an air/water mixture when accurate modelling of this type of breaker is sought.

The disadvantages of this technique are based on the estimation the velocity field only in the aerated fluid. This enables the use of the BIV technique in turbulent hydrodynamic conditions and ++places++ as a good complementary tool for cases where the fluid is highly aerated and the use of other methods is not possible.

\section{ACKNOWLEDGEMENT}

The research was supported in part by research grants from the National Autonomous University of Mexico (PAPIIT IN106610) and the National Council of Science and Technology in Mexico (CONACYT) Project number SEP-SEB 167003. 


\section{REFERENCES}

Elgar, S., Herbers, T. H. C., and Guza, R. T. (1994) Reflection of ocean surface gravity waves from a natural beach. J. Phys. Oceanogr., 24, 1503-1511

Govender, K., Mocke, G.P., and Alport M.J., 2004. Dissipation of isotropic turbulence and length-scale measurements through the wave roller in laboratory spilling waves. J Geophys Res. 109 (C08018):5115-5124

Hassan, Y.A., Schmidl, W.D., and Ortiz-Villafuerte, J. (1998) Investigation of three-dimensional two phase flow structures in a bubbly pipe. Meas. Sci Technol., 9, 309-326

Holland, K.T, Puleo, J.A., and Kooney, T.N. (2001) Quantification of swash flows using video-based particle image velocimetry. Coastal Engineering, 42(2), 65-77

Jayaratne, R., Hunt-Raby, A., Bullock, G., and Bredmose, H. (2008) Individual violent overtopping events: new insights. In: Proceedings of the 31 th international conference on coastal engineering, Hamburg, World Scientific., 1-13

Lindken, R., and Merzkirch, W. (2002) A novel PIV technique for measurements in multi-phase bubby flows. Exp. Fluids, 33, 814-825

Lin, P., and Liu, P.F. (1998a) A numerical study of breaking waves. J.Fluid Mech., 359, 239-264

Lin, P., and Liu, P.F. (1998b) Turbulent transport, vorticity dynamics and solute mixing under plunging waves in surf zones. J.Geopsh Res.,103(15), 15667-15964

Nishino, K., Kato, H., and Torii, K. (2000) Stereo imaging for simultaneous measurement of size and velocity of particles in dispersed two-phase flow. Meas. Sci Technol., 11, 633-645

Petti, M., Longo, S. (2001) Turbulence experiments in the swash zone. Coastal Engineering, 43, 1-24

Pedrozo-Acuña, A., Simmonds, D. J., and Reeve, D. E. (2008) Wave-impact characteristics of plunging breakers acting on gravel beaches. Mar. Geol. K, 253(1): 26-35

Ray, S.D. (2002) Applied photographic optics. Focal, Oxford, 215-233

Raubenheimber, B., Elgar, S., and Guza, R. T. (1996) Wave transformation across the inner surf zone. J. Geophys. Res., 101, 25.589-25.597.

Rivillas-Ospina, G. D., Pedrozo-Acuña, A., and Silva, R., and Gutierrez, C (2011) Estimation of the velocity field induced by plunging breakers in the surf zone. Exp. Fluids, 52, 53-68.

Ryu, Y., Chang, K.A., and Lim, H.J. (2005) Use of bubble image velocimetry for measurement of plunging wave impinging on structure and associated greenwater. Meas. Sci Technol., 16, 19451953

Ryu, Y., Chang K.A., and Mercier, R. (2007) Runup and green water velocities due to breaking waves impinging and overtopping. Exp. Fluids., 43, 555-567

Ryu, Y., and Chang K.A. (2008) Green water void fraction due to breaking wave impinging and overtopping. Exp. Fluids., 45, 883-898

Scott, C.P., Cox, D.T., Maddux, T.B., and Long, J. W. (2005) Large-scale laboratory observations of turbulence on a fixed barred beach. Meas. Sci. and Tech., 16, 1903-1912.

Ting, F.C.K., and Kirby J.T. (1994) Observation of undertow and turbulence in a laboratory surf zone. Coastal Engineering, 24(1-2), 51-80

Ting, F.C.K., and Kirby, J.T. (1995) Dynamics of surf-zone turbulence in a strong plunging breaker. Coastal Engineering, 24, 177-204

Ting, F.C.K., and Kirby, J.T. (1996) Dynamics of surf-zone turbulence in a strong plunging breaker. Coastal Engineering, 27, 131-160

Ting, F.C.K. (2006) Large-scale turbulence under a solitary wave. Coastal Engineering, 53(5-6), 441462 